\title{
Multimodal Anesthesia for Glaucoma Surgery in a Child with Mitochondrial Disease and Malignant Hyperthermia
}

\author{
Jacqueline Tutiven ${ }^{1}$, Benjamin Pruden ${ }^{2 *}$ and Howard Palte ${ }^{3}$ \\ ${ }^{1}$ Assistant Professor of Anesthesiology, Department Of Anesthesiology, Florida \\ ${ }^{2}$ Anesthesiology Resident, Jackson Memorial Hospital, Florida \\ ${ }^{3}$ Associate Professor of Anesthesiology, Department of Anesthesiology, Florida
}

Submission: November 22, 2016; Published: December 01, 2016

*Corresponding author: Benjamin Pruden, Anesthesiology Resident, Jackson Memorial Hospital, Miami, Florida, Tel: 317-501-0793,

Email: benjamin.pruden@jhsmiami.org

\begin{abstract}
Mitochondrial diseases (MD) are characterized by impairments of mitochondrial function that precipitate metabolic acidosis. An 8-year-old MD female with prior anesthesia exposure complicated by metabolic acidosis, seizures and propofol infusion syndrome, presented for glaucoma surgery. We present a multimodal balanced anesthetic technique used to successfully manage this complex case.
\end{abstract}

\section{Introduction}

Mitochondrial disorders (MD) are a heterogeneous group of genetic disorders that impair mitochondrial integrity and result in deficient energy production. The disorder has an incidence of 1:5000 live births [1], and affects tissues with high-energy requirements such as the central nervous system, retina, heart and muscle [2]. Consequently, these patients have multiple comorbidities that include cardiac, endocrine, and neurologic dysfunction [3-5]. Current evidence suggests that mitochondrial disease may be a contributing factor in the pathogenesis of glaucoma [6]. Anesthesia in this patient population may prove hazardous because the stress of surgery and fasting can induce marked metabolic aberrations, most commonly lactic acidosis [7].

\section{Case Presentation}

Institutional Review Board approval is not required for single case reports at Jackson Memorial Hospital and University of Miami. An 8 year old, $25 \mathrm{~kg}$ Caucasian female with infantile glaucoma, mitochondrial disease, renal tubular acidosis type II and family history of malignant hyperthermia presented for bilateral glaucoma surgery. Of note, at age 6, eye surgery under GA had been complicated by severe post-operative metabolic acidosis, propofol infusion syndrome (PRIS) and prolonged two week PICU stay. At that time, genetic investigation revealed an abnormality of the 2-oxoglutarate-dehydrogenase enzyme.

A multidisciplinary group comprising physicians from genetics, neurology, cardiology, nephrology and anesthesiology was convened and recommended new studies in order to identify the gene deficit. Also, the group recommended preoperative nutritional supplementation and electrolyte correction. Her ECG demonstrated prolongation of the QT interval (426ms) and the 2-D cardiac echo was normal. Current medications included trileptal, brimonidine and lantaprost ophthalmic solutions, coenzyme Q10 and a multivitamin supplement.

Our anesthesia plan centered on a balanced technique utilizing intravenous agents for sedation and hypnosis in tandem with extraconal ophthalmic block for analgesia. A vapor-free operating room was prepared. Monitoring included standard ASA monitors and bispectral analysis (BIS) (Model 185-0151) with the target range set at 40-60. In the holding suite the child was sedated with midazolam. Then, in the OR, infusions of remifentanil $(0.1 \mathrm{mcg} /$ $\mathrm{kg} / \mathrm{min})$ and dexmedetomidine $(1 \mathrm{mcg} / \mathrm{kg} / \mathrm{hr})$ were started. When BIS levels fell below 60 we administered cisatracurium $(4 \mathrm{mg})$ and performed endotracheal intubation. A left radial intraarterial catheter was placed for blood gas and metabolic panel 
analysis. Prior to surgery, a periorbital block using $2 \%$ lidocaine was performed.

Serial metabolic analysis demonstrated stable acid/base status with marginal elevations in serum lactic acid (1.3 to $1.5 \mathrm{mg} / \mathrm{dL}$ ). Surgery was completed in 110 minutes and passed uneventfully. At this point the neuromuscular block was antagonized, infusions were discontinued and the child was extubated and transferred to PICU for overnight observation. She was discharged from the hospital on postoperative day 1 .

\section{Discussion}

Mitochondrial disease (MD) are a divergent group of more than 100 genetic aberrations in which defects of the organelle impair oxidative phosphorylation and the production of energy, resulting in cell injury and metabolic acidosis. It is estimated that MD afflicts 1:5000 children. Symptoms typically manifest in tissues that are wholly dependent on mitochondria as a source of energy [8].

The anesthetic management of children with MD is complex because studies indicate that volatile inhalational agents depress mitochondrial function $[9,10]$. Additionally, severe co morbidities such as organ failure are potential risks for lactic acidosis. Further anesthesia considerations include sensitivity to neuromuscular blocking agents, altered homeostasis and impairment of mitochondrial integrity triggered by several intravenous agents. There are no controlled clinical trials to assess the effects of anesthetic agents and their correlation with intra- and postoperative complications [3]. Currently, available data stems from case reports, expert opinion and retrospective chart reviews $[10,11]$.

A recent rat model study demonstrated that ketamine produces alterations in the mitochondrial respiratory chain complex 1 [12]. Other investigations implicate etomidate and barbiturates as inhibitors of the same complex [13]. Moreover, evidence suggests that propofol impairs mitochondrial function, and that propofol infusions are associated with PRIS because this agent is highly lipophilic and readily diffuses across cell membranes. Therefore, propofol should only be administered as a single bolus $[14,15]$. On the other hand, dexmedetomidine, a selective $\alpha 2$ agonist, is known to have beneficial effects on the mitochondrial membrane [16]. Although the association between $\mathrm{MD}$ and malignant hyperthermia $(\mathrm{MH})$ remains unproven, the Malignant Hyperthermia Association of the United States (MHAUS) continues to recommend avoidance of volatile anesthetic agents and caution in using succinylcholine [17-19].

In this patient we were concerned about the development of lactic acidosis, malignant hyperthermia and PRIS. At the same time, we aimed to optimize intraoperative surgical conditions and avoid patient movement. In light of these considerations; we adopted a balanced intravenous anesthetic technique that eliminated the need for a volatile inhalational agent. Thus, we integrated the hypnotic effect of dexmedetomidine and the analgesic property of an ultra-short acting opioid (remifentanil) with a non-depolarizing neuromuscular blocking agent having negligible renal elimination (cisatracurium) and also performed an extraconal eye block (2\% lidocaine). We selected lidocaine because bupivacaine inhibits carnitine-acylcarnitine translocase and has been reported to precipitate ventricular dysrhythmias [20]. The eye block was quintessential because it reduced anesthetic requirements and assured a smooth transition between the operating room (OR) and post anesthesia care unit (PACU).

In summary, the anesthesia care for MD children is challenging. This case report highlights two important points; [1] the value of a multidisciplinary team to optimize preoperative status, and [2] the benefit of judicious selection of anesthetic technique in order to avoid complications, such as lactic acidosis, MH or PRIS. Finally, this case underscores the benefit of regional anesthesia in reducing anesthetic requirements and facilitating an uneventful transition from the operating room to post anesthesia care unit.

\section{References}

1. Koenig MK (2008) Presentation and diagnosis of mitochondrial disorders in children. Pediatr Neurol 38(5): 305-313.

2. Footitt EJ, Sinha MD, Raiman JA, Dhawan A, Moganasundram S, et al (2008) Mitochondrial disorders and general anaesthesia: a case series and review. Br J Anaesth 100(4): 436-441.

3. Maslow A, Lisbon A (1993) Anesthetic considerations in patients with mitochondrial dysfunction. Anesth Analg 76(4): 884-886.

4. Niezgoda J, Morgan P (2013) Anesthetic considerations in patients with Mitochondrial Defects. Pediatr Anesth 23(9): 785-793.

5. Rafique MB, Cameron SD, Khan Q, Biliciler S, Zubair S (2013) Anesthesia for children with mitochondrial disorders: A national survey and review. J Anesth 27(2): 186-191.

6. Kong, GY, Van Bergen NJ, Trounce IA, Crowston JG (2009) Mitochondrial dysfunction and glaucoma. J Glaucoma 18(2): 93-100.

7. Shipton EA, Prosser DO (2004) Mitochondrial myopathies and anaesthesia. Eur J Anaesthesiol 21 (3): 173-178.

8. Cohen PJ (1973) Effects of anesthetics on mitochondrial function. Anesthesiology 39(2): 153-164.

9. Miro O, Barrientos A, Alonso JR, Casademont J, Jarreta D et al. (1999) Effects of generalanaesthetic procedures on mitochondrial function of human skeletal muscle. Eur JClin Pharmacology 55(1): 35-41.

10. Miyamoto Y, Miyashita T, Takaki S, Goto T (2016) Perioperative considerations in adult mitochondrial disease: A case series and a review of 111 cases. Mitochondrion 26: 26-32.

11. Chow SY, Woon KL (2015) General anesthesia for adults with mitochondrial myopathy. A A Case Rep 4(5): 52-57.

12. Venancio C, Felix L, Almeida V, Coutinho J, Antunes L et al. (2015) Acute ketamine impairs mitochondrial function and promotes superoxide dismutase activity in ratbrain. Anesth Analg 120(2): 320-328.

13. Devin A, Nogueira V, Averet N, Leverve X (2006) Profound effects of generalanesthetic etomidate on oxidative phosphorylation without effects on their yield. J Bioenerg Biomemb 38(2): 137-142.

14. Fleisher LA (2005) Anesthesia and uncommon disease. ( $5^{\text {th }}$ edn), Elsevier, Philadelphia, USA, pp. 433-443.

15. Bains R, Moe MC, Vinje ML, Berg-Johnsen J (2009) Sevoflurane and propofol depolarizemitochondria in rat and human 
cerebrocorticalsynaptosomes by different mechanisms. Acta Anaesthesiol Scand 53(10): 1354-1360.

16. Engelhard K, Werner C, Kaspar S, Mollenberg O, Blobner M et al. ( 2002) Effects of the alpha2agonist dexmedetomidine on cerebral neurotransmitter concentrations during cerebral ischemia in rats. Anesthesiology 96(2): 450-457.

17. Al-Takrouri H, Martin TW, Mayhew JF (2004) Hyperkalemic cardiac arrest following succinylcholine administration: The use of extracorporeal membrane oxygenation in an emergency situation. J Clin Anesth. 16(6): 449-451.
18. Larach MG, Rosenberg H, Gronert GA, Allen GC (1997) Hyperkalemic cardiac arrest during anesthesia in infantsand children with occult myopathies. Clin Pedaitr (Phila) 36(1): 9-16.

19. Hopkins PM (2011) Malignant hyperthermia: pharmacology of triggering. Br J Anaesth 107(1): 48-56.

20. Weinberg GL, Palmer JW, VadeBoncouer TR, Zuechner MB, Edelman G (2000) Bupivicaine inhibits acylcarnitine exchange in cardiac mitochondria. Anesthesiology 92(2): 523-528. 